\title{
Evaluative conditioning of artificial grammars: Evidence that subjectively-unconscious structures bias affective evalua- tions of novel stimuli
}

\author{
Răzvan Jurchiş, Andrei Costea, Zoltan Dienes, Mircea Miclea, Adrian Opre
}

\begin{abstract}
Evaluative conditioning (EC) refers to the acquisition of emotional valence by an initially-neutral stimulus (conditioned stimulus; CS), after being paired with an emotional stimulus (unconditioned stimulus; US). An important issue regards whether, when participants are unaware of the CS-US contingency, the affective valence can generalize to new stimuli that share similarities with the CS. Previous studies have shown that generalization of EC effects appears only when participants are aware of the contingencies, but we suggest that this is because (a) the contingencies typically used in these studies are salient and easy to detect consciously, and (b) the performance-based measures of awareness (so-called "objective measures"), typically used in these studies, tend to overestimate the amount of available conscious knowledge. We report a preregistered study in which participants $(\mathrm{N}=217)$ were exposed to letter strings generated from two complex artificial grammars that are difficult to decipher consciously. Stimuli from one grammar were paired with positive USs, while those from the other grammar were paired with negative USs. Subsequently, participants evaluated new, previously-unseen, stimuli from the positively-conditioned grammar more positively than new stimuli from the negatively-conditioned grammar. Importantly, this effect appeared even when trial-by-trial subjective measures indicated lack of relevant conscious knowledge. We provide evidence for the generalization of EC effects even without subjective awareness of the structures that enable those generalizations.
\end{abstract}

\section{Preregsitration https://osf.io/cbdu6 Supplementary materials: https://osf.io/5jgwp/}

Keywords: evaluative conditioning; generalization; artificial grammar learning; implicit learning; emotion

Evaluative conditioning (EC) is defined as the acquisition of positive or negative affective valence by an initially-neutral stimulus (conditioned stimulus; CS), due to its co-occurrence with a positive or negative stimulus (unconditioned stimulus; US) (e.g., Levey \& Martin, 1975). Decades of research have shown that

Răzvan Jurchiș, Andrei Costea, Mircea Miclea, and Adrian Opre - Cognitive Psychology Laboratory, Department of Psychology, Babeș-Bolyai University, Romania

Zoltan Dienes - School of Psychology and Sackler Center for Consciousness Science, University of Sussex, UK

The present material has been posted as a preprint (https://psyarxiv.com/jkfu5/) and has been presented at the 8th Implicit Learning Seminar (Troms $\varnothing$, Norway, June 6th - 8th, 2019). The authors would like to thank Olivier Corneille, Anthony Greenwald, and three other anonymous reviewers for their efforts towards improving the paper.

Correspondence should be addressed to Răzvan Jurchiș: email: razvanjurchis@psychology.ro; postal address: Institute of Psychology, 37 Republicii Street 400015, Cluj-Napoca, Romania this process can influence evaluations of initially-neutral persons, social categories, brands, and various other types of stimuli (Hofmann et al., 2010).

Most EC studies try to change the affective valence only for the specific CSs that are paired with the USs. However, in order for EC to have a significant impact in daily life, the effect must also generalize to previously-unseen stimuli that share similarities with the presented CSs (Hütter \& Tigges, 2019; Unkelbach, Stahl, \& Forderer, 2012). Moreover, a core objective for EC research has been to determine whether the conditioning (e.g., Sweldens, Corneille, \& Yzerbyt, 2014) and the generalization (e.g., Glaser \& Kuchenbrandr, 2017) effects can appear without the awareness of the contingencies formed between the CSs and the USs, because evaluations that are based on unconscious knowledge might have different properties from consciously-based ones: For example, the former could be more difficult to change, to integrate with information from other sources (cf. Aust, Haaf, \& Stahl, 2019; Baars, 1997), to communicate to other persons (Mer- 
cier \& Sperber, 2018), to control or to prevent from influencing behavior (cf. Hütter, Sweldens, Unkelbach, \& Klauer, 2012; Jacoby, 1991).

In order to test whether generalization can occur without awareness, Glaser and Kuchenbrandt (2017) presented participants with two groups of alien creatures. On their heads, members of one group had an antenna, while members of the other group had a triangleshaped object. When members of one group were paired with negative (or positive) images, even previously-unseen creatures that had the same object on the head, were evaluated more negatively (or positively). Thus, this study has found evidence for generalization to new stimuli, but only when participants were aware of the contingencies (i.e., remembered accurately which group of creatures was associated with which valence; for similar results, see Hütter, Kutzner, \& Fiedler, 2014, and Hütter \& Tigges, 2019). In conclusion, generalization of EC effects to new stimuli seems possible, but the available evidence largely shows that generalization is dependent on awareness (contrast $\mathrm{Ol}$ son \& Fazio, 2006; see Glaser \& Kuchenbrandt, 2017, for a discussion).

We argue that, even if generalization of EC would be possible without awareness, most studies present participants with simple regularities that are easy to detect consciously: The CSs paired with a positive valence are easily distinguishable from those paired with negative valence [e.g., they belong to clearly different and salient categories (e.g., men versus women in Hütter et al., 2014) and/or are differentiated by obvious cues (e.g., Glaser \& Kuchenbrandt, 2017)]. Furthermore, these generalization studies typically use socalled "objective" measures of awareness, which assume that, if participants identify accurately the valence the CSs have been paired with, they have conscious memory of the CS-US contingencies. However, these methods are biased towards overestimating the role of awareness in conditioning and in generalization effects (e.g., Sweldens et al., 2014; Sweldens, Tuk, \& Hütter, 2017), because participants are able to identify accurately the valence from sources other than the conscious memories of the CS-US or CS-valence pairings: that is, from their affective reaction towards the CS (Hütter et al., 2012; Sweldens et al., 2014) and from implicit memory-based feelings of familiarity (Sweldens et al., 2017; Timmermans \& Cleeremans, 2015).

\footnotetext{
1 Although it has not been explicitly framed as a subjective method, the process-dissociation method developed by Hütter et al. (2012) is a subjective measure of awareness, since it requires participants to respond contingent on their own assessment of their mental states. That is, for each CS, they have to introspectively as-
}

While alternative, subjective, measures of awareness have already begun to gain dominance in the study of EC (especially the process-dissociation method of Hütter et al., 2012) ${ }^{1}$, these measures are more difficult to adapt to generalizations studies, so they have not penetrated the generalization literature. In sum, most previous studies on the generalization of EC (a) employed methods that favour the development of conscious knowledge, by using salient contingencies and, further, (b) used measures that are suspected to overestimate the amount of conscious knowledge available to participants.

In the present study, we propose an approach that employs more complex regularities that are difficult to detect consciously, but that are nonetheless learned, producing mostly unconscious knowledge. Also, starting from current theories of consciousness, such as Global Workspace and Higher-Order Thought theories, we use a subjective measure of awareness, which can be more specific in disentangling implicit from explicit influences (e.g., Dienes, 2012, LeDoux \& Hofmann, 2018; contrast Shanks, 2005), if specific conditions regarding the sensitivity, relevance, immediacy and reliability of the measure are met (Berry \& Dienes, 1993; Shanks \& St John, 1994; Sweldens et al., 2014, 2017).

The regularities that we use are called artificial grammars, and consist of complex sets of rules that specify permitted, "grammatical", combinations of elements (Figure 1). In a typical Artificial Grammar Learning task (AGL; Reber, 1967), participants are presented with meaningless letter strings that, unknown to them, follow an artificial grammar. After exposure, participants are informed that the strings followed some rules, but nothing is disclosed about the rules' configuration. Then, they are presented with new strings, some of which follow the same grammar, some of which don't. Participants' task is to respond, for every string, whether it follows the grammar or not. The typical result is that participants are able to classify accurately new strings as "grammatical" or "non-grammatical", even when they rely on subjectively-unconscious knowledge of the grammar (e.g., Dienes \& Scott, 2005; Ivanchei \& Moroskina, 2018; Norman \& Price, 2012; Scott \& Dienes, 2008, 2010; contrast Shanks, 2005; although the task typically also produces some conscious knowledge). Moreover, several studies have shown that participants can learn two grammars, and classify new

sess whether they have explicit memory for the CS-valence association. In the Exclusion task, if they remember consciously the CSvalence association, they have to respond contrary to their memory of the CS-valence association; if they do not remember the valence, they have to respond according to their attitude towards the CS. 
strings according to any of the two grammars (e.g., Dienes, Altmann, Kwan, \& Goode, 1995; Norman, Scott, Price, \& Dienes, 2016).

\section{The Present Study.}

We propose a task in which, in an acquisition/conditioning phase, strings generated according to one grammar are paired with negative stimuli, while strings generated according to the other grammar are paired with positive stimuli. For brevity, we call the grammar associated with negative stimuli "the negative grammar", and the grammar paired with positive stimuli, "the positive grammar". We assume that participants will acquire structures coding the relations between the elements of the grammars, and between the elements of established CS-US or CS-valence relations, the evaluated strings in our task can be made more or less pleasant by the relations between grammatical elements in their composition and a valence (e.g., between the fragment RTV and a positive valence). Note that, even if participants learned some elements of the grammar (e.g, that TV appeared after R), if they did not also learn that those elements were related with a valence, the generalized EC effect could not appear. In conclusion, we are interested, for each string, whether participants are aware of the relations between elements of the grammar and the valence, which make the string more or less pleasant. Participants can be unaware of these relations for two reasons: First, because they are unaware of the grammar, hence they have no conscious

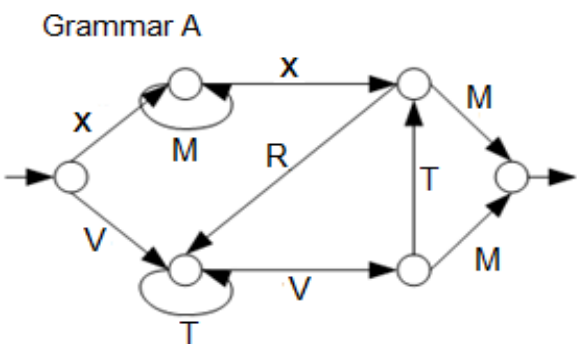

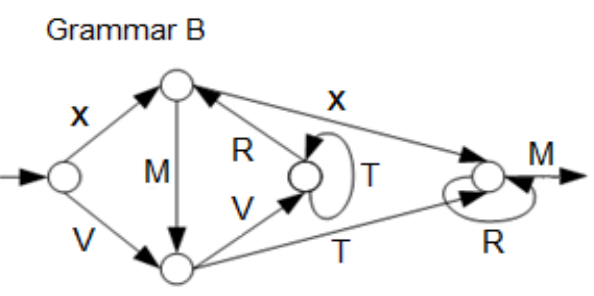

Figure 1. The artificial grammars used in the present study (Dienes et al., 1995; Reber, 1967; Norman et al., 2016). Strings are generated by following the order permitted by the arrows. For example, XMXRTVM is consistent only with grammar A and XMVTRXM is consistent only with grammar $B$.

the grammar and the positive/negative affect. Accordingly, in a subsequent test phase, we expect that participants will evaluate new strings that follow the "positive grammar" more positively, compared to new strings that follow the "negative grammar". In this case, it means that we have obtained a generalizable conditioning effect, because participants evaluate previously-unseen strings.

EC research is typically interested whether conditioning effects occur when participants do not remember consciously (a) the specific US the evaluated CS has been paired with and/or (b) the valence the evaluated CS has been paired with (e.g., Sweldens et al., 2014). In contrast, in our task, participants do not evaluate CSs that have been directly paired with a US or with a valence; they evaluate stimuli that contain elements from a grammar that has been associated with a valence. Therefore, while in standard EC and EC generalization studies the CSs are made more or less pleasant by the

\footnotetext{
${ }^{2}$ These two reasons are analogous to the reasons for unawareness from typical EC and EC generalization studies: Participants could be unaware of the CS-valence relations either because (1) they cannot remember consciously whether the CS has been presented in the
}

content to consciously relate with the valence. Second, because, even if they are aware of the grammar, they are unaware of whether it was paired with a positive or a negative affect. $^{2}$

If we detect an EC effect in the absence of awareness, this would mean, more specifically, that participants generalize their evaluations towards new stimuli without being aware of the relations between the elements of the grammar and the affective valence, which enable them to make those generalizations.

Our conceptualization and measure of awareness is based on Global availability/global workspace (Baars, 1997; Dienes, 2012; Shea \& Frith, 2019), and, even closer, on Higher-order thoughts theories of consciousness (e.g., Rosenthal, 2005), which assume that, in order to be aware of a mental content, one has to have the representation that one has the content (see Dienes, 2008a, 2012; Dienes \& Scott, 2005). Accordingly, we probe the existence of these meta-representations that are necessary for consciousness, by asking participants

conditioning phase, thus they have no conscious content to consciously relate with a valence or (2) they remember having seen the $\mathrm{CS}$, but cannot remember whether it was paired with positive or with negative affect. 
to report what they know about their knowledge of the structures coding the relations between elements of the grammar and a valence, that make the string likable or dislikeable. If participants exhibit, objectively, knowledge of the structures (inferred from the differential liking of strings that follow the positive versus the negative grammar) when they claim that they do not have the knowledge, it means that their knowledge of the structures is unconscious (cf., e.g., Dienes, 2012; Dienes \& Scott, 2005; Ling et al., 2018; Seth, Dienes, Cleeremans, Overgaard, \& Pessoa, 2008). We use a subjective measure of awareness, since we require participants to discriminate between their own mental states. Although subjective measures of awareness have been previously criticized in the context of EC research, it was typically not their subjective character, per se, that has been criticized. Rather, the target of the criticism has been the relatively low sensitivity of the specific scales used. For instance, they probed the presence of conscious knowledge via open-ended questions, which were presented only at the end of the experiment. Hence, participants might underreport conscious knowledge (e.g., Sweldens et al., 2014). As presented in the Method section (and in Supplementary C), our measure of awareness differs substantially from the subjective measures previously used in EC research, and is specifically tailored to address limitations of past measures, in the context of our task.

We hypothesized that (i) there will be an overall conditioning effect, (ii) a conditioning effect based on unconscious knowledge and, also, (iii) a conditioning effect based on conscious knowledge. Furthermore, we compared the unconscious with the conscious effects, in order to determine (iv) whether awareness enhances the generalization of the EC.

\section{Method}

The hypotheses, procedure, data collection, and statistical analyses were preregistered here: https://osf.io/cbdu6/

\section{Participants}

A number of 240 undergraduate students from a Romanian university underwent the task, in exchange for course credit; 23 failed the attention/engagement checks (see Procedure), therefore the final sample is composed of 217 participants ( 176 women, $\mathrm{m}_{\mathrm{age}}=19.94$ years, $S D=4.14)$.

\section{Materials}

We employed the two grammars depicted in Figure 1 , and took the strings from Norman et al. (2016). In the acquisition/conditioning phase, we presented 32 strings from each grammar. In the test phase, participants had to evaluate 20 strings from each grammar. In the acquisition phase, the strings from one grammar were always presented together with negative images, while the strings from the other grammar were always presented with positive images. For counterbalancing, for some randomly-determined participants, grammar A was the positive grammar (and grammar B the negative one); conversely for the rest of participants. We used 23 positive and 23 negative images, taken from IAPS (Lang et al., 1997), NAPS (Marchewka et al., 2014), and OASIS (Kurdi et al., 2017) (see Supplementary A for the list of images and strings). It was randomly determined which particular image appeared with which particular string.

\section{Procedure}

The study was conducted according to the regulations of Babeș-Bolyai University's Research committee and with the APA Ethical guidelines. All participants gave written consent. Data were collected online, using gorilla.sc (Anwyl-Irvine et al., 2019). All instructions and response options were presented in Romanian (the original Romanian version and a translated English version are presented in Supplementary A).

Acquisition/conditioning phase. In this phase, participants were expected to learn both grammars and to associate them with emotions. On each trial, the emotional image appeared first. Then, after 1.5 seconds, the string appeared just below the image. The string remained on the screen, together with the image, for 7.5 seconds, then the next trial began automatically. Participants were instructed to memorize which string appears together with which image. They were not informed that there are regularities in how the strings were constructed or that they would have to do a subsequent affective evaluation task.

The acquisition phase was divided in four blocks: two for grammar A and two for grammar B. After each block, participants had a 30 seconds break. In each block, participants were exposed to all 32 strings from one grammar, each string being paired with an emotional image. Thus, in each block, the participant saw strings that followed only one grammar and images that had only one valence (positive or negative), in order to facilitate learning the grammar, by reducing interference with the other grammar and to benefit from emotional carry-over effects. It was randomly determined 
whether the participant was exposed first to the grammar associated with the negative affect or to that associated with the positive affect.

At ten random moments in the acquisition, we presented, for attention/engagement check purposes, a string and an image, and asked participants whether the string and the image they saw in that moment were identical or not with those from the previous trial. If both were identical, the correct answer was "Yes". If either the string or the image was changed, the correct answer was "No". Based on the calibration from a pilot study, we excluded participants that made more than four mistakes.

Test phase (valence rating and awareness measure). Participants were presented with new letter strings, without images. They had to evaluate, for every string, how much they like or dislike it, using a Likert scale with values ranging from -5 (strongly dislike) to +5 (strongly like). The scale did not have a neutral point (i.e., zero was removed) because while piloting the task, participants reported that it felt strange to rate preference for meaningless strings of letters, and had the tendency to give neutral ratings. Therefore, by removing the neutral point, we intended to constrain participants to attend even very subtle positive or negative feelings towards the strings (see Eder, Krishna, \& Van Dessel, in press, for a similar solution). After rating the string's valence, participants had to respond to an awareness scale, while the string was still on the screen. More specifically, we informed them that they might not know what makes some strings likeable/dislikeable, but that it is also possible that for some strings they might be aware of some "groups or patterns of letters" that make the strings likeable/dislikeable. The scale was adapted from awareness scales that are widely-used in AGL (e.g., Dienes \& Scott, 2005; Norman et al., 2016; Wierzchon et al., 2012) and in reward learning/operant conditioning studies (e.g., LeganesFonteneau, Scott, \& Duka, 2018):

To what extent are you aware of what makes the string likeable/dislikeable for you?

1- I don't have any clue about what makes it likeable/dislikeable

2- I I more or less guessing what makes it likeable/dislikeable, but I could not describe what it is that makes it likeable/dislikeable

\footnotetext{
${ }^{3}$ One reviewer suggested that participants might be aware of these relations, but that they might not be aware of their influence. This is unlikely, since it would mean that a participant, for example, rates positively the string XMXRTVM, is aware that the fragment
}

3- I think I know what makes it likeable/dislikeable

4- I know what makes it likeable/dislikeable

Since the evaluation of a string could be influenced by relations between a valence and elements of the grammar that were present in that string, if participants are aware of these relations, they should be able to identify, in each string, the grammatical elements that carry the affective valence and that make the string pleasant/unpleasant. ${ }^{3}$

Responses 1 and 2 denote that participants are not aware of the structures that make the string likeable/dislikeable, while responses 3 and 4 indicate that participants have some conscious knowledge of the structures (cf. Dienes \& Scott, 2005; Ramsøy \& Overgaard, 2004; Leganes-Fonteneau et al., 2018; Wierzchon et al., 2012). In order to decrease the probability that participants would report as unaware trials in which they actually used conscious knowledge, but held with low confidence, we explicitly instructed them to use response 3 if they have some conscious knowledge, even if they do not have much confidence in it (see Supplementary C for more details regarding the relevance, sensitivity, and reliability of the scale).

Finally, we collected data regarding age, sex, education, and socio-economic status. More specific details regarding the Stimuli and the Procedure are presented in Supplementary A and in the preregistration form (https://osf.io/cbdu6/).

\section{Results}

We report both Bayes factors and significance tests; our conclusions will follow from the Bayes factors, though in all cases both approaches lead to the same conclusions. In the Bayesian analyses, by convention we interpret Bayes factors $(B)$ between 0.33 and 3 as insensitive, $B$ between 3 and 10 as providing moderate evidence, and $B \geq 10$ as providing strong evidence for the alternative hypothesis (H1) over H0. Conversely, we interpret $1 / 3 \leq B<1 / 10$ as moderate evidence, and $B \leq 1 / 10$ as strong evidence for $\mathrm{H} 0$ over $\mathrm{H} 1$.

For testing the first three hypotheses, the model of $\mathrm{H} 1 \mathrm{used}$ in the Bayesian analyses was a half-normal with the mode of zero and the $S D$ equal to an expected raw effect size of 0.66 (accordingly, the Bayes factor is

RTV has been paired with a positive valence, has been informed that liking/disliking can be generated by groups/patterns of letters, but still responds s/he has no clue why s/he rated positively a string containing the fragment RTV. 
Table 1.

Mean evaluative ratings received by strings from the positive and the negative grammar, split on different levels of awareness

\begin{tabular}{lccccc}
\hline & All responses & $\mathbf{1 \& 2}$ & $\mathbf{1}$ & $\mathbf{2}$ & $\mathbf{3 \& 4}$ \\
& $(\mathrm{N}=217)$ & $(\mathrm{N}=209)$ & $(\mathrm{N}=193)$ & $(\mathrm{N}=176)$ & $(\mathrm{N}=182)$ \\
\hline Positive grammar & $0.153(1.036)$ & $-0.279(1.166)$ & $-0.543(1.370)$ & $0.109(1.575)$ & $1.218(1.857)$ \\
Negative grammar & $-0.386(1.119)$ & $-0.596(1.120)$ & $-0.788(1.287)$ & $-0.196(1.686)$ & $0.155(2.103)$ \\
\hline
\end{tabular}

Note. The values represent means and, those in parentheses, standard deviations. Ns refer to the number of participants that used a specific awareness level with strings from both the positive and the negative grammar and that were included in the analyses. Ns differ because not all participants had responses for all awareness levels. Numbers on the top row refer to awareness levels: 1 - I don't have any clue...; 2 - I am more or less guessing...; 3- I think I know...; 4 - I know... .

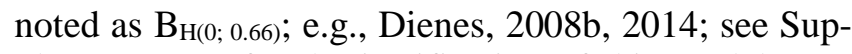
plementary B for the justification of this model, preregistered before data collection). Typically, we would report robustness regions for Bayes factors, but in what follows the results are so clear there was no need.

In order to determine whether there was an overall conditioning effect, we compared the ratings received by all strings that followed the positive grammar with those received by the strings that followed the negative one, irrespective of the conscious/unconscious basis of the evaluation. Table 1 presents mean evaluative ratings received by strings from negative and from positive grammars. A repeated-measures $t$-test revealed, as expected, that strings from the positive grammar were evaluated more positively than those from the negative grammar, $t(216)=7.69, p<.00001$, Cohen's $d_{z}=0.52$, $m_{\text {diff }}=0.538,95 \%$ CI $[0.40 ; 0.67], \mathrm{B}_{\mathrm{H}(0 ; 0.66)}=10^{12}$.
As in typical AGL studies (Dienes \& Scott, 2005), most participants' responses $(66.05 \%)$ were based on unconscious knowledge (i.e., responses 1 and 2 at the awareness scale). When testing whether there was a conditioning effect based on unconscious knowledge (Figure 2A), we found, again, that the strings from the positive grammar were evaluated more positively than those from the negative grammar, $t(208)=4.38, p=$ $.00001, d_{z}=0.30, m_{\text {diff }}=0.317,95 \%$ CI $[0.17 ; 0.46]$, $\mathrm{B}_{\mathrm{H}(0 ; 0.66)}=3,133.45$.

We found the same pattern when we analyzed separately responses 1 and 2 . For response $1: t(192)=2.34$, $p=.004, d_{z}=0.20, m_{\mathrm{diff}}=0.245,95 \% \mathrm{CI}[0.06 ; 0.43]$, $\mathrm{B}_{\mathrm{H}(0 ; 0.66)}=9.53$. For response $2: t(175)=2.67, p=.01$, $d_{z}=0.17, m_{\mathrm{diff}}=0.305,95 \% \mathrm{CI}[0.05 ; 1.39], \mathrm{B}_{\mathrm{H}(0 ; 0.66)}=$ 5.41. The effect was also present when participants relied on conscious knowledge (responses 3 and 4 from

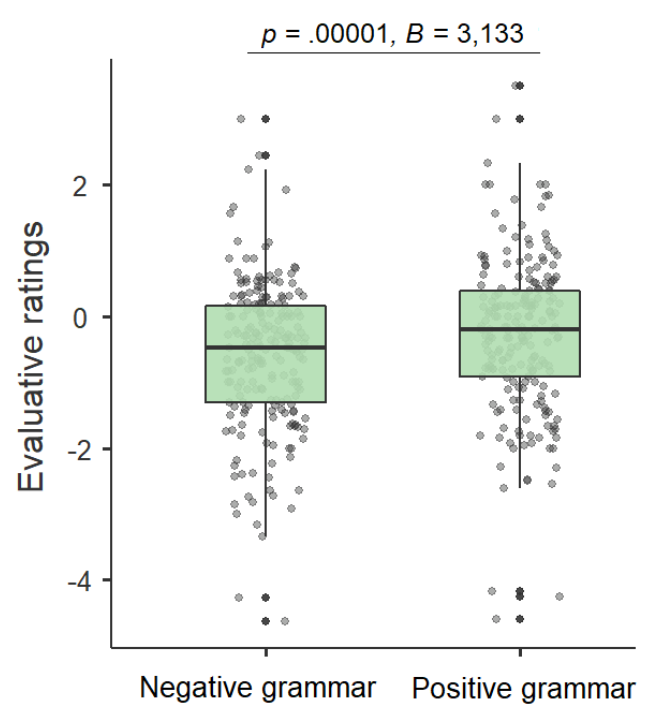

(A) Unconscious knowledge

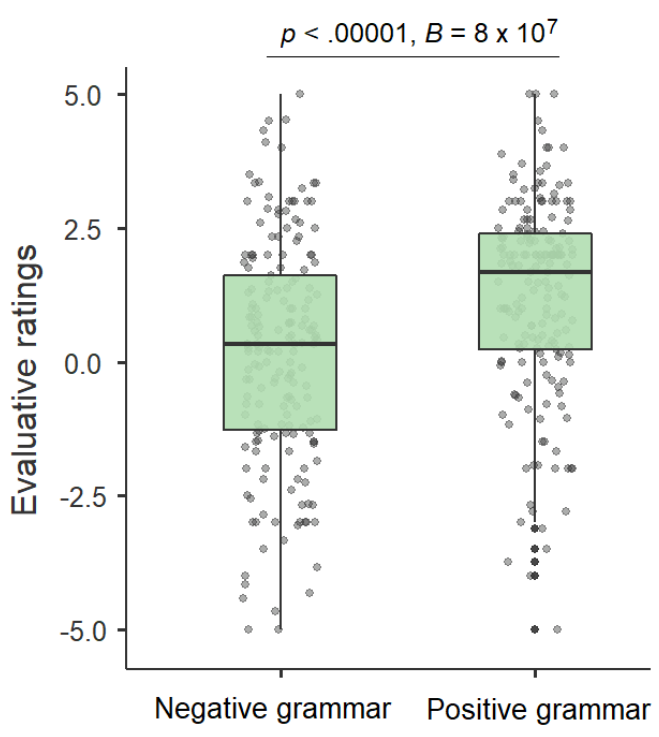

(B) Conscious knowledge

Figure 2. Evaluative ratings received by strings from the positive and the negative grammars when participants used (A) unconscious knowledge and (B) conscious knowledge. 
the awareness scale; Figure $2 \mathrm{~B}), t(181)=6.35, p<$ $.00001, d_{z}=0.47, m_{\mathrm{diff}}=1.06,95 \% \mathrm{CI}[0.73 ; 1.39], \mathrm{B}_{\mathrm{H}(0 ;}$ $0.66)=8 \times 10^{7}$. Finally, we found that the conditioning effect based on conscious knowledge was higher than that based on unconscious knowledge, $t(173)=4.60, p$ $<.00001, d_{z}=0.35, m_{\text {diff }}=0.822,95 \%$ CI $[0.47 ; 1.18]$, $\mathrm{B}_{\mathrm{U}(0 ; 1.59)}=11,993$. This Bayesian test was conducted with a different prior (see Supplementary B).

In sum, we found strong support for (i) an overall conditioning effect, (ii) an effect based on unconscious knowledge, (iii) an effect based on conscious knowledge, and (iv) that the conscious effect was higher than the unconscious one.

\section{Discussion}

The present study is one of the first to show evidence for EC of complex structures. Moreover, it offers evidence that the EC effect generalizes to new stimuli that follow the conditioned structures, even in the absence of subjective awareness of the structures, contradicting previous studies that found affective processing only in the presence of awareness (e.g., Lähteenmäki, Hyönä, Koivisto, \& Nummenmaa, 2015). However, consistent with the previous literature, the effect was significantly stronger in the presence of conscious knowledge, supporting the idea that awareness is an important moderator of EC (e.g., Hofmann et al., 2010).

While we believe that the present study can contribute to the discussions regarding the role of awareness in EC and, more broadly, in affective phenomena, it departs substantially from the existing approaches in EC research: First, we did not condition individual stimuli, but structures followed by stimuli. Consequently, a second difference was that we did not measure whether participants remembered consciously the valence the evaluated strings were paired with (as they were not paired directly with any US), but we evaluated whether participants were aware of the structures (i.e., of the contingencies between elements of the grammars and valence) that make each string likeable/dislikeable. Third, we subscribed to a subjective definition and measurement of awareness, while most previous studies on the relationship between EC and awareness have used objective measurements. In sum, we consider that the present study provides evidence for a new phenomenon in the family of EC effects, where participants like or dislike a previously unseen stimulus, because the stimulus follows an emotionally-loaded structure that participants are subjectively-unaware of.

\section{Limitations}

Even though we took several measures for ensuring that our assessment of awareness is reliable and sensitive, it still has several limitations: First, we cannot rule out the possibility that participants inferred, post-hoc, parts of the regularities that were associated with positive and with negative affect. For instance, in the test phase, they could notice that a specific group of letters always triggers negative affect. Hence, after observing this relationship, they responded that they know what makes the string negative. Therefore, even though conscious knowledge did not play a role in their evaluation (as it emerged post-evaluation), this would count, according to our scale, as an evaluation based on conscious knowledge. This limitation could lead to an underestimation of the unconscious EC effect, because some evaluations sustained by unconscious knowledge would be attributed to conscious knowledge (cf, e.g., Sweldens et al., 2014).

Second, it is possible that our awareness measure did not disentangle all types of knowledge that could sustain participants' evaluations. Specifically, unconscious knowledge could produce generalized EC effects based on two routes. Let's assume that a participant has to evaluate the string XMXRTVM, which follows the positive grammar, and gives it a positive rating. First, this could be because the participant holds an unconscious association, say, between the trigram RTV and the positive valence. In this case, the participant holds no conscious knowledge of the association between elements of the grammar and the emotion, so she should use level 1 of the awareness scale (I don't have any clue...). In a second case, the participant has learned unconsciously something about the grammar's configuration (e.g., TV appeared after R), and, based on her unconscious knowledge of the grammar's configuration (TV after R), she might be able to judge consciously that the string is somehow similar to the strings paired with positive images (even though she doesn't know what makes it similar to those strings; e.g., Dienes \& Scott, 2005; Norman \& Price, 2012). In these situations, participants would be likely to use level 3 of the awareness scale (I think I know...) but, since they cannot fully express the reasons for liking (they do not know what makes the string belong to those paired with positive images), they could also use level 2 (I am more or less guessing...). Even in this case, the fact that the conscious judgment of similarity was based on unconscious knowledge of the grammars, enables us to draw the conclusion that the detected EC effect was influenced by non-conscious structures (see, for more details, the distinction between structural and judgment 
knowledge from the AGL literature; Dienes \& Scott, 2005). Moreover, the effect appeared even when we excluded responses based on level 2 of the scale, and took into consideration only evaluations based on response 1 (I don't have any clue about what makes it likeable/dislikeable), which was designed to exclude any type of conscious knowledge. ${ }^{4}$

Also, the AGL task has been criticized on the grounds that participants can discriminate strings from the two grammars not necessarily by acquiring complex structures, but by identifying bigrams and trigrams (i.e., successions of two or three letters) that differ between the grammars. Furthermore, since these bigrams and trigrams are relatively simple, they are relatively easy to be detected consciously, thus they are sometimes represented explicitly (cf. Dulany, Carlson, \& Dewey, 1984; Perruchet \& Pacteau, 1990). However, several studies from the past decades, using various implicit learning tasks, have found that even when the regularities are solely at the level of bigrams and trigrams, most of participants' knowledge is still implicit (e.g., Fu, Dienes, \& Fu, 2012; Gomez, 1997; Ling et al., 2018). Thus, it is unwarranted to assume that if participants learn bigrams and trigrams, learning is, ipso facto, conscious. In the same vein, all previous AGL studies that have used the very same grammars and the very same acquisition and test strings that we used, consistently found evidence for implicit learning (Dienes et al., 1995; Wan, Dienes, \& Fu, 2008; Norman, Price, \& Jones, 2011; Norman et al., 2016, 2019). Finally, we conducted additional (non-preregistered) analyses on our data which show that, while liking is indeed predicted by bigrams and trigrams, it is also predicted by more complex and abstract types of knowledge, which are, presumably, more difficult to be consciously detected (see Supplementary B).

\section{Implications and future directions}

A propositional account would have difficulties in fully explaining a conditioning effect when participants have no conscious knowledge of the structures present in a string, since the formation of propositions is assumed to be applicable only to conscious contents (e.g., De Houwer, 2018). For situations discussed above, in which participants were, presumably, aware that the string is somehow related to the strings paired with a valence, but were not aware of the grammar elements

\footnotetext{
${ }^{4}$ We consider very unlikely a more extreme scenario, in which participants evaluated the string as pleasant because it was similar to the strings paired with positive images, and were also aware of the elements that make it is similar to the positively-conditioned strings, but systematically responded that they have no idea what
}

that gave this similarity, relating the string with a valence could be explained by the formation of conscious propositions (e.g., since the string is more similar to those paired with positive valence, the string is pleasant/likeable). However, the acquisition of unconscious structures, which were responsible for ensuring the similarity, cannot be explained by the formation of propositions, but rather by the formation of associations between the elements of the grammar. Cases where participants lacked any kind of conscious knowledge leave even less room for an influence of propositions, but, again, these situations might be explained by the unconscious formation of associations between elements of the grammar, and between elements of the grammar and a valence (e.g., Greenwald \& De Houwer, 2017; see Corneille \& Stahl, 2019, for a discussion). More precise data on the conscious/unconscious status of all types of knowledge acquired in this task (knowledge of the grammars, knowledge of the grammar-valence contingencies) would also enable more precise discussions regarding the nature of the representations responsible for the detected generalized EC effect.

Beyond the EC literature, our results are also compatible with those from research on affect misattribution (e.g., Schwarz \& Clore, 1983, 2003; but see Simonsohn, 2014 and Cummins, Hussey, \& Hughes, 2019), which has claimed that participants are not always able to identify accurately what influences their affective responses. For example, they can attribute their negative affect to a low life satisfaction, even though the negative affect was actually generated by a rainy weather (Schwarz \& Clore, 1983). Thus, in these studies, participants exhibit affective influences from stimuli they are aware of (e.g., they are aware that the weather is rainy), while being unaware of their influence (i.e., think something else exerts the influence). Our study presents a particular case of incapacity to consciously detect the causes for one's affective evaluations, in which the causes are newly-acquired, conditioned, structures, and in which the influence is generalized to previously-unseen stimuli that fit those structures. Moreover, in the present study, the causes themselves (i.e. the associations between elements of the

makes the string positive. This scenario would invalidate our conclusion, that nonconscious structures had an influence on the EC effect, but given the measures we took for ensuring the validity of our awareness scale, we consider it highly implausible. 
grammar and a valence) were unaware, not only their influence. $^{5}$

Future studies should try to assess awareness for different components that can contribute to the detected EC effect: In addition to awareness of the relations between elements of the grammar and the valence, they should also assess awareness that a string belongs to the positively- or to the negatively-conditioned grammar and awareness of the grammar elements that makes the string belong to that grammar. This would help to determine whether, as explained above, the combination of unconscious knowledge of the grammars but with conscious knowledge of the similarity (Dienes \& Scott, 2005), is indeed involved in producing the EC effect.

Future studies could also investigate additional characteristics of the revealed EC effect, such as its automaticity or controllability. For instance, in affectivepriming tasks, stimuli with emotional valence automatically enhance the detection of stimuli with the same valence (Fazio, Sanbonmatsu, Powell, \& Kardes, 1986; Ivanchei \& Asvarich, 2018). Using the present paradigm, participants might undergo an affective-priming task in which primes could be new strings that follow the positive or the negative grammar. Another approach would be to adopt process-dissociation methods, as those often used in AGL (e.g., Norman et al., 2016) and EC research (e.g., Hütter et al., 2014), and to test whether participants can voluntarily control the influence of the acquired structures.

Other essential properties regard the malleability of consciously- and unconsciously-based EC effects. It would be important to determine whether extinction of the EC effect is achieved in different time frames when the effect is sustained by conscious versus unconscious knowledge; or whether, for example, there are differences in the amount of exposure needed to positively condition a structure that has been initially conditioned negatively, as a function of conscious versus unconscious status of knowledge or of the associative versus propositional representations sustaining the EC effect (cf. Mann, Kurdi, \& Banaji, 2019).

In conclusion, the present study proposes a novel experimental paradigm in EC research, in which evaluations are based on structures, provides evidence that

\footnotetext{
${ }^{5}$ When the real causes are conscious (e.g., participants are aware that the weather is rainy), the misattribution effect is claimed to appear only when participants' attention is actively directed, via experimental manipulation, towards the mistaken cause (e.g., they are asked explicitly about their life satisfaction, but no mention is made about the weather). When the manipulation hints, even subtly, to the real causes (e.g., by asking participants about the weather), the misattribution effect is claimed to disappear (see also Cummins et
}

EC effects can generalize in the absence of subjective awareness of the conditioned structures, and, more generally, that subjectively non-conscious structures can influence affective responses.

\section{Context of the Research}

We are often able to evaluate whether a sentence is grammatically correct or incorrect, even without being able to explain why it is so. Thus, our evaluations can be based on complex non-conscious knowledge about word associations permitted by a certain language. Can knowledge that we are unaware of also influence our affective evaluations? We show in this study that we can learn very complex associations between neutral stimuli and positive or negative emotions, and that these associations can remain outside awareness, but still influence our affective judgments.

For research on unconsciously-based affective phenomena, this approach based on learning complex associations presents advantages over existing methods: it permits the use of strong, supraliminal, stimuli (as opposed to the methods using subliminal exposure), and, also, it keeps participants unaware of the regularities in most of the trials (as opposed to some of the studies using simple CS-US relations). Accordingly, while not without limitations (e.g., it doesn't afford a direct manipulation of awareness, as in the case of subliminal exposure), it could complement existing approaches in the study of unconscious emotional learning.

We are interested to test in future studies whether unconscious evaluative and fear conditioning based on such complex structures can occur in more ecologically-relevant contexts, with regards to more realistic stimuli (e.g., with regards to complex behaviours of a virtual avatar).

al., 2019). In contrast, in our task, participants' attention was actively directed towards the real causes for the evaluation (i.e., were told that the affective valence can be carried by groups or patterns of letters), thus we have little reason to suspect that in trials in which participants claimed to have no conscious knowledge, they were actually aware of the structures and were unaware only of their influence. 


\section{References}

Anwyl-Irvine, A. L., Massonnié, J., Flitton, A., Kirkham, N., \& Evershed, J. K. (2019). Gorilla in our Midst: An online behavioral experiment builder. Behavior Research Methods. Doi: https://doi.org/10.3758/s13428-019-01237-x

Aust, F., Haaf, J. M., \& Stahl, C. (2019). A memory-based judgment account of expectancy-liking dissociations in evaluative conditioning. Journal of Experimental Psychology: Learning, Memory, and Cognition, 45(3), 417-439. Doi: http://dx.doi.org/10.1037/xlm0000600

Baars, B. J. (1997). In the Theatre of Consciousness. The Workspace of the Mind. New York, NY: Oxford University Press.

Bradley, M. M., Codispoti, M., Sabatinelli, D., \& Lang, P. J. (2001). Emotion and motivation II: sex differences in picture processing. Emotion, 1(3), 300-319.

Brooks, L. R., \& Vokey, J. R. (1991). Abstract analogies and abstracted grammars: Comments on Reber (1989) and Mathews et al. (1989). Journal of Experimental Psychology: General, 120(3), 316-323.

Corneille, O., \& Stahl, C. (2019). Associative attitude learning: A closer look at evidence and how it relates to attitude models. Personality and Social Psychology Review, 23(2), 161-189. Doi: https://doi.org/10.1177/1088868318763261

Cummins, J., Hussey, I., \& Hughes, S. (2019, May 21). The AMPeror's New Clothes: Performance on the Affect Misattribution Procedure is Mainly Driven by Awareness of Influence of the Primes. Doi: https://doi.org/10.31234/osf.io/d5zn8

Dienes, Z. (2008a). Subjective measures of unconscious knowledge. Progress in Brain Research, 168, 49-64.

Dienes, Z. (2008b). Understanding Psychology as a Science: An Introduction to Scientific and Statistical Inference. New York, NY: Palgrave Macmillan.

Dienes, Z. (2012). Conscious versus unconscious learning of structure. In P. Rebuschat \& J. Williams (Eds), Statistical Learning and Language Acquisition. Mouton de Gruyter Publishers (pp. 337 - 364).

Dienes, Z. (2014). Using Bayes to get the most out of non-significant results. Frontiers in Psychology, 5, 781. Doi: https://doi.org/10.3389/fpsyg.2014.00781

Dienes, Z., Altmann, G., Kwan, L., \& Goode, A. (1995). Unconscious knowledge of artificial grammars is applied strategically. Journal of Experimental Psychology: Learning, Memory, and Cognition, 21(5), 1322-1338. Doi: http://dx.doi.org/10.1037/0278-7393.21.5.1322

Dienes, Z., \& Scott, R. (2005). Measuring unconscious knowledge: Distinguishing structural knowledge and judgment knowledge. Psychological Research, 69(5-6), 338-351. Doi: https://doi.org/10.1007/s00426-004-0208-3

De Houwer, J. (2018). Propositional models of evaluative conditioning. Social Psychological Bulletin, 13(3), e28046. Doi: https://doi.org/10.5964/spb.v13i3.28046

Dulany, D. E., Carlson, R. A., \& Dewey, G. I. (1984). A case of syntactical learning and judgment: How conscious and how abstract? Journal of Experimental Psychology: General, 113(4), 541-555. doi:10.1037/0096-3445.113.4.541

Eder, A. B., Krishna, A., \& Van Dessel, P. (in press). Operant Evaluative Conditioning. Journal of Experimental Psychology: Animal Learning and Cognition

Fazio, R. H., Sanbonmatsu, D. M., Powell, M. C., \& Kardes, F. R. (1986). On the automatic activation of attitudes. Journal of Personality and Social Psychology, 50(2), 229-238. Doi: http://dx.doi.org/10.1037/0022-3514.50.2.229
Fu, Q., Dienes, Z., \& Fu, X. (2010). Can unconscious knowledge allow control in sequence learning?. Consciousness and Cognition, 19(1), 462-474.

Glaser, T., \& Kuchenbrandt, D. (2017). Generalization effects in evaluative conditioning: Evidence for attitude transfer effects from single exemplars to social categories. Frontiers in Psychology, 8, 103. https://doi.org/10.3389/fpsyg.2017.00103

Gomez, R. L. (1997). Transfer and complexity in artificial grammar learning. Cognitive Psychology, 33(2), 154-207.

Greenwald, A. G., \& De Houwer, J. (2017). Unconscious conditioning: Demonstration of existence and difference from conscious conditioning. Journal of Experimental Psychology: General, 146(12), 1705-1721. Doi: http://dx.doi.org/10.1037/xge0000371

Hofmann, W., De Houwer, J., Perugini, M., Baeyens, F., \& Crombez, G. (2010). Evaluative conditioning in humans: a metaanalysis. Psychological Bulletin, 136(3), 390-421. Doi: http://dx.doi.org/10.1037/a0018916

Hütter, M., Kutzner, F., \& Fiedler, K. (2014). What is learned from repeated pairings? On the scope and generalizability of evaluative conditioning. Journal of Experimental Psychology: General, 143(2), 631-643. Doi: http://dx.doi.org/10.1037/a0033409

Hütter, M., Sweldens, S., Stahl, C., Unkelbach, C., \& Klauer, K. C. (2012). Dissociating contingency awareness and conditioned attitudes: Evidence of contingency-unaware evaluative conditioning. Journal of Experimental Psychology: General, 141(3), 539-557.

Hütter, M., \& Tigges, D. (2019). On the external validity of evaluative conditioning: Evaluative responses generalize to modified instances of conditioned stimuli. Journal of Experimental Social Psychology, 84, 103824. https://doi.org/10.1016/j.jesp.2019.103824

Ivanchei, I. I., \& Asvarisch, A. (2018). The Nature Of Affect In The Structural Mere Exposure Effect. National Research University Higher School of Economics, Working Papers, Series: Psychology (No. WP BRP 99/PSY/2018).

Ivanchei, I. I., \& Moroshkina, N. V. (2018). The effect of subjective awareness measures on performance in artificial grammar learning task. Consciousness and Cognition, 57, 116-133. Doi: https://doi.org/10.1016/j.concog.2017.11.010

Jacoby, L. L. (1991). A process dissociation framework: Separating automatic from intentional uses of memory. Journal of Memory and Language, 30(5), 513-541. Doi: https://doi.org/10.1016/0749-596X(91)90025-F

Jurchiş, R., Costea, A., Dienes, Z., Mircea, M., \& Opre, A. (2019, November 13). Evaluative conditioning of artificial grammars (Supplementary information and preregistration). Retrieved from osf.io/5jgwp

Kurdi, B., Lozano, S., \& Banaji, M. R. (2017). Introducing the open affective standardized

image set (OASIS). Behavior Research Methods, 49(2), 457-470. Doi: https://doi.org/10.3758/s13428-016-0715-3

Lähteenmäki, M., Hyönä, J., Koivisto, M., \& Nummenmaa, L. (2015). Affective processing requires awareness. Journal of Experimental Psychology: General, 144(2), 339-345. Doi: http://dx.doi.org/10.1037/xge0000040

Lang, P. J., Bradley, M. M., \& Cuthbert, B. N. (1997). International affective picture system

(IAPS): Technical manual and affective ratings. NIMH Center for the Study of Emotion and Attention, 39-58. 
LeDoux, J. E., \& Hofmann, S. G. (2018). The subjective experience of emotion: a fearful view. Current Opinion in Behavioral Sciences, 19, 67-72. Doi: https://doi.org/10.1016/j.cobeha.2017.09.011

Leganes-Fonteneau, M., Scott, R., \& Duka, T. (2018). Attentional responses to stimuli associated with a reward can occur in the absence of knowledge of their predictive values. Behavioural Brain Research, 341, 26-36. Doi: https://doi.org/10.1016/j.bbr.2017.12.015

Levey, A. B., \& Martin, I. (1975). Classical conditioning of human 'evaluative' responses. Behaviour Research and Therapy, 13(4), 221-226. Doi: https://doi.org/10.1016/00057967(75)90026-1

Ling, X., Zheng, L., Guo, X., Li, S., Song, S., Sun, L., \& Dienes, Z. (2018). Cross cultural differences in implicit learning of chunks versus symmetries. Royal Society Open Science, 5, doi: https://doi.org/10.1098/rsos.180469

Lotz, A., \& Kinder, A. (2006). Transfer in artificial grammar learning: The role of repetition information. Journal of Experimental Psychology: Learning, Memory, and Cognition, 32(4), 707-715.

Marchewka, A., Żurawski, Ł., Jednoróg, K., \& Grabowska, A. (2014). The Nencki Affective

Picture System (NAPS): Introduction to a novel, standardized, wide-range, high-quality, realistic picture database. Behavior Research Methods, 46(2), 596-610. Doi: https://doi.org/10.3758/s13428-013-0379-1

Mann, T. C., Kurdi, B., \& Banaji, M. R. (2019). How effectively can implicit evaluations be updated? Using evaluative statements after aversive repeated evaluative pairings. Journal of Experimental Psychology: General. $h$ ttps://doi.org/10.1037/xge0000701

Mercier, H., \& Sperber, D. (2018). The Enigma of Reason. Cambridge, MA: Harvard University Press.

Meulemans, T., \& Van der Linden, M. (1997). Associative chunk strength in artificial grammar learning. Journal of Experimental Psychology: Learning, Memory, and Cognition, 23(4), 1007 1028. doi:10.1037/0278-7393.23.4.1007.

Mierop, A., Hütter, M., \& Corneille, O. (2017). Resource availability and explicit memory largely determine evaluative conditioning effects in a paradigm claimed to be conducive to implicit attitude acquisition. Social Psychological and Personality Science, 8(7), 758-767.

Newell, B. R., \& Shanks, D. R. (2014). Unconscious influences on decision making: A critical review. Behavioral and Brain Sciences, 37(1), 1-19. Doi: 10.1017/S0140525X12003214

Norman, E., \& Price, M. C. (2012). Social intuition as a form of implicit learning: Sequences of body movements are learned less explicitly than letter sequences. Advances in Cognitive Psychology, 8(2), 121-131. doi: 10.2478/v10053-008-0109-x

Norman, E., Price, M. C., \& Jones, E. (2011). Measuring strategic control in artificial grammar learning. Consciousness and Cognition, 20, 1920-1929. Doi: https://doi.org/10.1016/j.con$\operatorname{cog} .2011 .07 .008$

Norman, E., Scott, R. B., Price, M. C., \& Dienes, Z. (2016). The relationship between strategic control and conscious structural knowledge in artificial grammar learning. Consciousness and Cognition, 42, 229-236. https://doi.org/10.1016/j.concog.2016.03.014

Norman, E., Scott, R. B., Price, M. C., Jones, E., \& Dienes, Z. (2019). Can unconscious structural knowledge be strategically controlled? In Cleeremans, A., Allakhverdov, V., \& Kuvaldina, M. (Eds), Implicit learning: 50 years on. Routledge (pp 159-173).
Olson, M. A., \& Fazio, R. H. (2006). Reducing automatically activated racial prejudice through implicit evaluative conditioning. Personality and Social Psychology Bulletin, 32(4), 421-433. Doi: https://doi.org/10.1177/0146167205284004

Paciorek, A., \& Williams, J. N. (2015). Semantic generalization in implicit language learning. Journal of Experimental Psychology: Learning, Memory, and Cognition, 41(4), 989-1002.

Perruchet, P., \& Pacteau, C. (1990). Synthetic grammar learning: Implicit rule abstraction or explicit fragmentary knowledge?. Journal of Experimental Psychology: General, 119(3), 264 275. doi:10.1037/0096-3445.119.3.264

Ramsøy, T. Z., \& Overgaard, M. (2004). Introspection and subliminal perception. Phenomenology and the Cognitive Sciences, $3(1), 1-23$. https://doi.org/10.1023/B:PHEN.0000041900.30172.e8

Reber, A. S. (1967). Implicit learning of artificial grammars. Journal of Verbal Learning and Verbal Behavior, 6(6), 855-863. https://doi.org/10.1016/S0022-5371(67)80149-X

Rosenthal, D. M. (2004). Varieties of higher-order theory. Advances in Consciousness Research, 56, 17-44.

Schwarz, N., \& Clore, G. L. (1983). Mood, misattribution, and judgments of well-being: Informative and directive functions of affective states. Journal of Personality and Social Psychology, 45(3), 513-523.

Schwarz, N., \& Clore, G. L. (2003). Mood as information: 20 years later. Psychological inquiry, 14(3-4), 296-303. Doi: https://doi.org/10.1080/1047840X.2003.9682896

Scott, R. B., \& Dienes, Z. (2008). The conscious, the unconscious, and familiarity. Journal of Experimental Psychology: Learning, Memory, and Cognition, 34(5), 1264-1288. http://dx.doi.org/10.1037/a0012943

Scott, R. B., \& Dienes, Z. (2010). Knowledge applied to new domains: The unconscious succeeds where the conscious fails. Consciousness and Cognition, 19(1), 391-398. http://dx.doi.org/10.1037/a0012943

Seth, A. K., Dienes, Z., Cleeremans, A., Overgaard, M., \& Pessoa, L. (2008). Measuring consciousness: relating behavioural and neurophysiological approaches. Trends in Cognitive Sciences, 12(8), 314-321. Doi: https://doi.org/10.1016/j.tics.2008.04.008

Shanks, D. R. (2005). Implicit learning. In K. Lamberts and R. Goldstone (Eds.), Handbook of Cognition (pp. 202-220). London, UK: Sage.

Shea, N., \& Frith, C. D. (2019). The Global Workspace Needs Metacognition. Trends in Cognitive Sciences, 560-571. Doi: https://doi.org/10.1016/j.tics.2019.04.007

Simonsohn, U. (2015). Small telescopes: Detectability and the evaluation of replication results. Psychological Science, 26(5), 559-569. Doi: https://doi.org/10.1177/0956797614567341

Stahl, C., \& Unkelbach, C. (2009). Evaluative learning with single versus multiple unconditioned stimuli: The role of contingency awareness. Journal of Experimental Psychology: Animal Behavior Processes, 35(2), 286-291.

Stahl, C., Unkelbach, C., \& Corneille, O. (2009). On the respective contributions of awareness of unconditioned stimulus valence and unconditioned stimulus identity in attitude formation through evaluative conditioning. Journal of Personality and Social Psychology, 97(3), 404-420. doi: 10.1037/a0016196

Sweldens, S., Corneille, O., \& Yzerbyt, V. (2014). The role of awareness in attitude formation through evaluative conditioning. Personality and Social Psychology Review, 18(2), 187209. Doi: https://doi.org/10.1177/1088868314527832 
Sweldens, S., Tuk, M. A., \& Hütter, M. (2017). How to study consciousness in consumer research, A commentary on Williams and Poehlman. Journal of Consumer Research, 44(2), 266-275. Doi: https://doi.org/10.1093/jcr/ucx044

Timmermans, B., \& Cleeremans, A. (2015). How can we measure awareness? An overview of current methods. In M. Overgaard (Ed.), Behavioural methods in consciousness research (pp. 2146). Oxford: Oxford University Press. doi: 10.1093/acprof:oso/9780199688890.003.0003
Unkelbach, C., Stahl, C., \& Förderer, S. (2012). Changing CS features alters evaluative responses in evaluative conditioning. Learning and Motivation, 43(3), 127-134. Doi: https://doi.org/10.1016/j.lmot.2012.04.003

Wan, L., Dienes, Z., \& Fu, X. (2008). Intentional control based on familiarity in artificial grammar learning. Consciousness and Cognition, 17(4), 1209-1218. https://doi.org/10.1016/j.concog.2008.06.007

Wierzchoń, M., Asanowicz, D., Paulewicz, B., \& Cleeremans, A. (2012). Subjective measures of consciousness in artificial grammar learning task. Consciousness and Cognition, 21(3), 1141-1153. http://dx.doi.org/10.1037/a0012943 